\title{
Real-World Comparative Effectiveness, Safety, and Health Care Costs of Oral Anticoagulants in Nonvalvular Atrial Fibrillation Patients in the U.S. Department of Defense Population
}

\begin{abstract}
Kiran Gupta, PhD; Jeffrey Trocio, MPH; Allison Keshishian, MPH; Qisu Zhang, MPH; Oluwaseyi Dina, MPH; Jack Mardekian, PhD; Lisa Rosenblatt, MD; Xianchen Liu, MD, PhD; Shalini Hede, PharmD;
\end{abstract} Anagha Nadkarni, PhD; and Tom Shank, PharmD

\begin{abstract}
BACKGROUND: The ARISTOTLE trial demonstrated that apixaban had significantly lower rates of stroke/systemic embolism (SE) and major bleeding than warfarin; however, no direct clinical trials between apixaban and other direct oral anticoagulants (DOACs) are available. Few real-world studies comparing the effectiveness and safety between DOACs have been conducted.
\end{abstract}

OBJECTIVE: To compare effectiveness, safety, and health care costs among oral anticoagulants (OACs) for nonvalvular atrial fibrillation (NVAF) patients in the U.S. Department of Defense (DoD) population.

METHODS: Adult NVAF patients initiating warfarin or DOACs (apixaban, rivaroxaban, and dabigatran) were selected from U.S. DoD data from January 1,2013 , to September 30,2015 . The first OAC claim date was designated as the index date. Patients initiating another OAC were matched 1:1 to apixaban patients using propensity score matching to balance demographics and clinical characteristics. Cox proportional hazards models were used to estimate the risk of stroke/SE and major bleeding for each $O A C$ versus apixaban. Generalized linear and two-part models with bootstrapping were used to compare all-cause health care costs and stroke/ SE-related and major bleeding-related medical costs.

RESULTS: Of the 41,001 eligible patients, 7,607 warfarin-apixaban, 4,129 dabigatran-apixaban, and 11,284 rivaroxaban-apixaban pairs were matched. Warfarin $(\mathrm{HR}=1.84 ; 95 \% \mathrm{Cl}=1.30-2.59 ; P<0.001)$ and rivaroxaban ( $\mathrm{HR}=1.46 ; 95 \% \mathrm{Cl}=1.08-1.98 ; P=0.015)$ were associated with a significantly higher risk of stroke/SE compared with apixaban. Dabigatran ( $\mathrm{HR}=1.17 ; 95 \% \mathrm{Cl}=0.68-2.03 ; P=0.573$ ) was associated with a numerically higher risk of stroke/SE compared with apixaban. Warfarin $(\mathrm{HR}=1.53$; $95 \% \mathrm{Cl}=1.24-1.89 ; P<0.001)$, dabigatran $(\mathrm{HR}=1.76 ; 95 \% \mathrm{Cl}=1.27-2.43$; $P<0.001)$, and rivaroxaban $(\mathrm{HR}=1.59 ; 95 \% \mathrm{Cl}=1.34-1.89 ; P<0.001)$ were associated with higher risks of major bleeding compared with apixaban.

Compared with apixaban, patients prescribed warfarin incurred numerically higher all-cause total health care costs per patient per month (PPPM) $(\$ 2,498$ vs. $\$ 2,277 ; P=0.148)$ and significantly higher stroke/SE-related ( $\$ 118$ vs. $\$ 46 ; P=0.012$ ) and major bleeding-related ( $\$ 166$ vs. $\$ 76$; $P=0.003)$ medical costs. Dabigatran patients incurred numerically higher all-cause total health care PPPM costs $(\$ 2,372$ vs. $\$ 2,143 ; P=0.150)$ and stroke/SE-related medical costs ( $\$ 61$ vs. $\$ 32 ; P=0.240$ ) but significantly higher major bleeding-related costs ( $\$ 114$ vs. $\$ 58 ; P=0.025)$. Rivaroxaban patients incurred significantly higher all-cause total health care costs $(\$ 2,546$ vs. $\$ 2,200 ; P<0.001)$ and major bleeding-related medical costs PPPM ( $\$ 137$ vs. $\$ 69 ; P<0.001$ ) but numerically higher stroke/SE-related medical costs PPPM ( $\$ 58$ vs. $\$ 38 ; P=0.057$ ).

CONCLUSIONS: Among NVAF patients in the U.S. DoD population, warfarin and rivaroxaban were associated with a significantly higher risk of stroke/ SE and major bleeding compared with apixaban. Dabigatran use was associated with a numerically higher risk of stroke/SE and a significantly higher risk of major bleeding compared with apixaban. Warfarin and dabigatran incurred numerically higher all-cause total health care costs compared with apixaban. Rivaroxaban was associated with significantly higher allcause total health care costs compared with apixaban.

J Manag Care Spec Pharm. 2018;24(11):1116-25

Copyright $\odot 2018$, Academy of Managed Care Pharmacy. All rights reserved.

\section{What is already known about this subject}

Warfarin has been the standard oral anticoagulant for decades; several direct oral anticoagulants were approved for stroke prevention in atrial fibrillation patients in recent years.

A prospective randomized clinical trial demonstrated that apixaban had significantly lower rates of stroke/systemic embolism (SE) and major bleeding than warfarin.

\section{What this study adds}

In the U.S. Department of Defense population, apixaban initiation was associated with a significantly lower risk of stroke/SE and major bleeding compared with warfarin and rivaroxaban. Apixaban initiation was associated with a lower risk of stroke/SE that was not statistically significant but was also associated with significantly lower risk of major bleeding compared with dabigatran initiation.

Compared with apixaban initiators, all-cause health care costs were significantly higher for rivaroxaban but similar for dabigatran and warfarin initiators.

A trial fibrillation (AF), the most common cardiac arrhythmia, is associated with a nearly 5-fold excess risk of stroke. ${ }^{1,2}$ In the United States in 2010, the estimated prevalence of AF was approximately 5.2 million. ${ }^{3}$ The annual direct expenses for nonvalvular AF (NVAF) were estimated at approximately $\$ 7$ billion, which included $\$ 3$ billion to $\$ 4$ billion for hospitalizations. ${ }^{4}$

The American College of Cardiology/American Heart Association/Heart Rhythm Society Guideline recommends oral anticoagulants (OACs) for patients with NVAF and previous stroke, transient ischemic attack, or $\mathrm{CHA}_{2} \mathrm{DS}_{2}$-VASc score 
$\geq 2 .{ }^{5}$ Adjusted-dose warfarin has been used prophylactically as an oral anticoagulant to reduce the risk of stroke among NVAF patients. ${ }^{6}$ In recent years, 4 direct OACs (DOACs; dabigatran, rivaroxaban, apixaban, and edoxaban) have been approved by the U.S. Food and Drug Administration for the prevention of stroke and systemic embolism (SE) in NVAF patients. These DOACs offer the advantages of fewer drug-drug interactions, an absence of major dietary effects, no requirement for regular international normalized ratio monitoring, and less risk of intracranial bleeding when compared with warfarin. ${ }^{6}$

In the phase 3 randomized clinical trials comparing the effectiveness and safety among DOACs and warfarin, DOAC patients had at least noninferior risks of stroke/SE than warfarin patients; however, risks of major bleeding varied between DOACs and warfarin. ${ }^{7-10}$ No direct clinical trials have compared dabigatran, rivaroxaban, and apixaban.

In addition to clinical trials, retrospective observational studies have also examined the comparative effectiveness and safety among OACs. ${ }^{11,12}$ A recent meta-analysis showed that apixaban was associated with a similar risk of stroke/SE versus warfarin, dabigatran, rivaroxaban, and edoxaban, as well as a lower risk of major bleeding compared with warfarin and rivaroxaban. ${ }^{12}$ Several studies have reported that apixaban patients incurred lower health care costs (inpatient and/or outpatient) compared with warfarin and rivaroxaban, but incurred similar costs compared with dabigatran. ${ }^{13-15}$ However, additional large real-world studies are warranted to support these results.

The U.S. Department of Defense (DoD) health care system is one of the largest health care plans in the United States. To the best of our knowledge, this is the first study using this dataset to examine the risk of stroke/SE and major bleeding, as well as health care costs, among apixaban and other OACs. The purpose of this study was to provide additional information on clinical and economic outcomes of OAC-naive patients newly prescribed apixaban, dabigatran, rivaroxaban, or warfarin in a real-world clinical practice setting.

\section{Methods}

\section{Data Source}

We conducted a retrospective analysis using the U.S. DoD data from January 1, 2012, to September 30, 2015. The DoD provides health care to more than 9.4 million beneficiaries located in all 50 U.S. states and multiple countries. Beneficiaries include active duty, activated Guard and Reserve, retirees, survivors, some inactive Guard and Reserve, and their family members. ${ }^{16}$ Most beneficiaries are retired service members and their family members (4.8 million, 51\%), many of whom are Medicare eligible. The data include information about the inpatient, outpatient, emergency room, and pharmacy claims from U.S. DoD facilities and civilian/private sector care for eligible beneficiaries.

\section{Patient Selection}

Treatment-naive patients with $\geq 1$ pharmacy claim for warfarin, apixaban, rivaroxaban, or dabigatran from January 1 , 2013, through September 30, 2015, were selected. Given the later market entry (and hence small sample size), edoxaban was not included in the study. The first DOAC prescription date was designated as the index date if patients had DOAC claims; the first warfarin prescription date was designated as the index date for patients without any DOAC claim. Patients were required to have continuous health plan enrollment for 12 months before the index date (baseline period) and $\geq 1$ medical claim for AF (International Classification of Diseases, Ninth Revision, Clinical Modification [ICD-9-CM] code 427.31) during the baseline period. ${ }^{17}$

Patients were excluded from the study if they had claims for valvular heart disease, heart valve replacement, or heart surgery; dialysis, kidney transplant, or end-stage chronic kidney disease; venous thromboembolism or reversible AF (pericarditis, hyperthyroidism, or thyrotoxicity); a pharmacy claim for an OAC during the baseline period; hip or knee replacement within 6 weeks before the index date; $>1$ OAC claim on the index date; or indication of pregnancy during the study period.

Patient demographics, clinical risk scores (Charlson Comorbidity Index [CCI], $\mathrm{CHA}_{2} \mathrm{DS}_{2}-\mathrm{VASc}$, and HAS-BLED), comorbidities, comedications, and hospitalizations during the 12-month baseline period were measured. Patient data were assessed from the day after the index date until the date of discontinuation, switch to an OAC other than the index drug, death, interruption in continuous enrollment (no gap was allowed), or the end of the study period (September 30, 2015), whichever occurred first. Discontinuation was defined as no evidence of an index OAC prescription for 30 days from the last day of supply of the last filled prescription. The discontinuation date was defined as the last day of supply of the last filled prescription. Switching was defined as a prescription for an OAC other than the index OAC prescription within 30 days before or after the discontinuation date. ${ }^{18}$

\section{Outcome Measures}

The primary outcomes were stroke/SE, major bleeding, and health care costs (all-cause, stroke/SE-related, and major bleeding-related). Stroke/SE and major bleeding were defined by the primary or secondary diagnosis position of inpatient claims. Stroke/SE included ischemic stroke, hemorrhagic stroke, and SE. Major bleeding included bleeding at key sites including-but not limited to-intracranial, gastrointestinal, liver, splenic, and ocular hemorrhage. The stroke/SE and major bleeding code lists were based on previously published studies and validated administrative claims-based algorithms..$^{19,20}$ 
Real-World Comparative Effectiveness, Safety, and Health Care Costs of Oral Anticoagulants

in Nonvalvular Atrial Fibrillation Patients in the U.S. Department of Defense Population

\section{FIGURE 1 Attrition Flowchart}

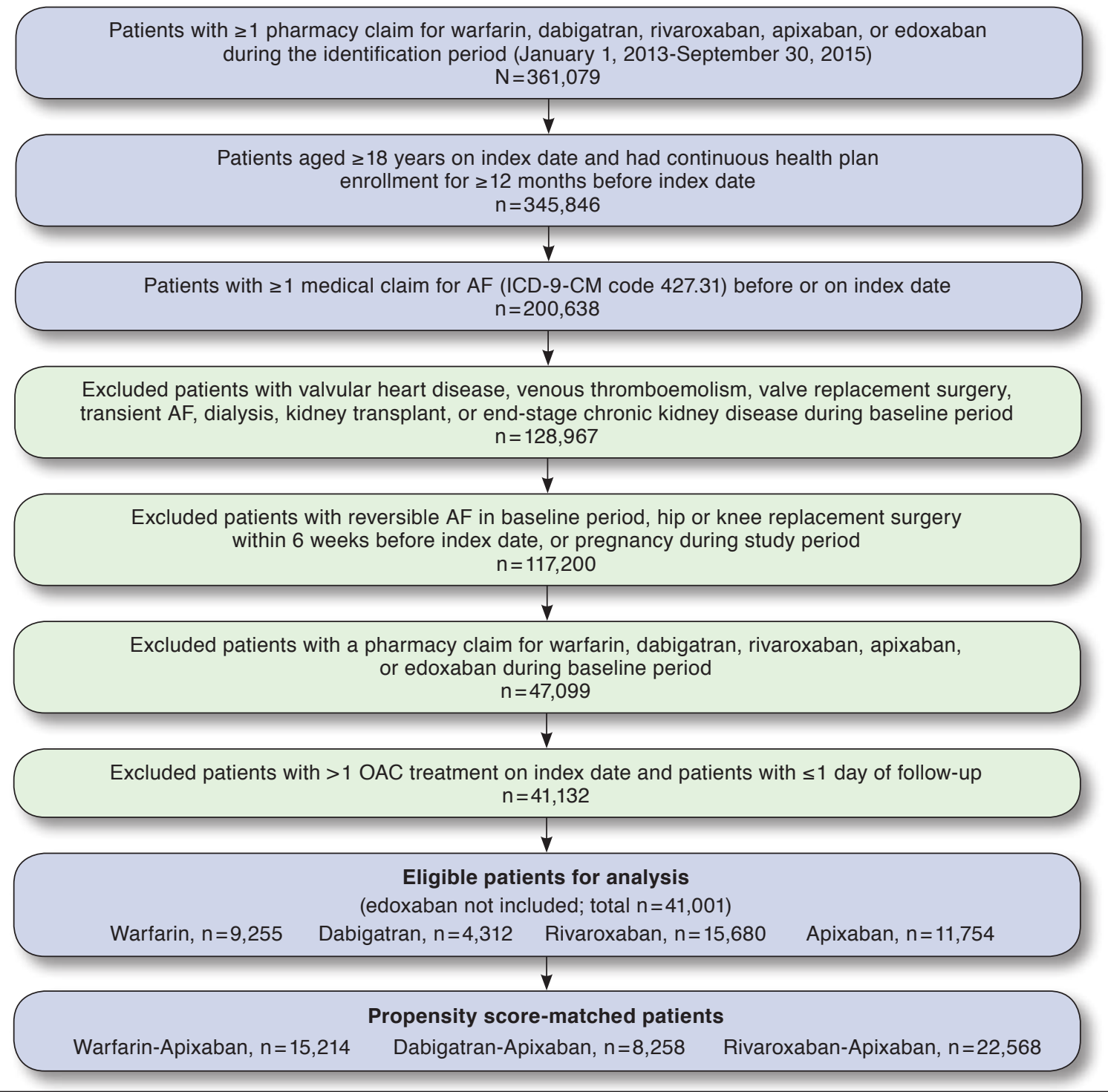

$A F=$ atrial fibrillation; ICD-9-CM = International Classification of Diseases, Ninth Revision, Clinical Modification; OAC=oral anticoagulant.

All-cause medical costs represent the sum of outpatient, emergency room, and inpatient costs. Total health care costs represent the sum of medical and pharmacy costs. Patients with insurance through the DoD do not have to pay for prescriptions filled through the DoD system; therefore, there is no cost value for those prescriptions. The pharmacy costs observed in this study were from TRICARE Mail Order Pharmacy and retail pharmacies. Patients pay copays for prescriptions filled through mail order or a traditional retail pharmacy.

Stroke/SE-related and major bleeding-related medical costs were defined as the hospitalization costs associated with the first stroke/SE or major bleeding event plus all subsequent stroke/SE or major bleeding costs occurring in the inpatient or outpatient setting. Per patient per month (PPPM) costs were measured and adjusted to 2015 U.S. dollars using the medical care component of the Consumer Price Index.

\section{Statistical Methods}

The design, analytical methods, and presentation of this study were informed by the guidelines for comparative effectiveness research. ${ }^{21,22}$ All study variables, including baseline and outcome measures, were analyzed descriptively and stratified by cohort. 


\section{Real-World Comparative Effectiveness, Safety, and Health Care Costs of Oral Anticoagulants in Nonvalvular Atrial Fibrillation Patients in the U.S. Department of Defense Population}

\section{TABLE 1 Baseline Characteristics of NVAF Patients in the Propensity Score-Matched Cohorts}

\begin{tabular}{|c|c|c|c|c|c|c|c|c|c|c|c|c|c|c|c|}
\hline & \multicolumn{2}{|c|}{$\begin{array}{c}\text { Apixaban } \\
\text { Cohort } \\
(\mathrm{n}=7,607)\end{array}$} & \multicolumn{2}{|c|}{$\begin{array}{c}\text { Warfarin } \\
\text { Cohort } \\
(\mathrm{n}=7,607)\end{array}$} & \multirow[b]{2}{*}{$\mathrm{STD}^{\mathrm{a}}$} & \multicolumn{2}{|c|}{$\begin{array}{c}\text { Apixaban } \\
\text { Cohort } \\
(\mathrm{n}=4,129)\end{array}$} & \multicolumn{2}{|c|}{$\begin{array}{c}\text { Dabigatran } \\
\text { Cohort } \\
(\mathrm{n}=4,129)\end{array}$} & \multirow[b]{2}{*}{$\mathrm{STD}^{\mathrm{a}}$} & \multicolumn{2}{|c|}{$\begin{array}{c}\text { Apixaban } \\
\text { Cohort } \\
(\mathrm{n}=11,284)\end{array}$} & \multicolumn{2}{|c|}{$\begin{array}{c}\text { Rivaroxaban } \\
\text { Cohort } \\
(\mathrm{n}=11,284)\end{array}$} & \multirow[b]{2}{*}{ STD $^{a}$} \\
\hline & n/Mean & $\% / S D$ & n/Mean & $\% / S D$ & & n/Mean & $\% / S D$ & n/Mean & $\% / S D$ & & n/Mean & $\% / S D$ & n/Mean & $\% / S D$ & \\
\hline Age & 76.5 & 9.5 & 76.6 & 9.8 & 0.95 & 73.0 & 10.0 & 73.0 & 9.9 & 0.05 & 75.3 & 9.6 & 75.3 & 9.5 & 0.12 \\
\hline $18-54$ & 172 & 2.3 & 178 & 2.3 & 0.53 & 183 & 4.4 & 160 & 3.9 & 2.79 & 315 & 2.8 & 293 & 2.6 & 1.20 \\
\hline $55-64$ & 558 & 7.3 & 563 & 7.4 & 0.25 & 523 & 12.7 & 561 & 13.6 & 2.73 & 993 & 8.8 & 1,007 & 8.9 & 0.44 \\
\hline $65-74$ & 2,157 & 28.4 & 2,057 & 27.0 & 2.94 & 1,472 & 35.7 & 1,487 & 36.0 & 0.76 & 3,518 & 31.2 & 3,493 & 31.0 & 0.48 \\
\hline$\geq 75$ & 4,720 & 62.0 & 4,809 & 63.2 & 2.42 & 1,951 & 47.3 & 1,921 & 46.5 & 1.46 & 6,458 & 57.2 & 6,491 & 57.5 & 0.59 \\
\hline \multicolumn{16}{|l|}{ Sex } \\
\hline Male & 4,431 & 58.2 & 4,430 & 58.2 & 0.03 & 2,551 & 61.8 & 2,538 & 61.5 & 0.65 & 6,431 & 57.0 & 6,411 & 56.8 & 0.36 \\
\hline Female & 3,176 & 41.8 & 3,177 & 41.8 & 0.03 & 1,578 & 38.2 & 1,591 & 38.5 & 0.65 & 4,853 & 43.0 & 4,873 & 43.2 & 0.36 \\
\hline \multicolumn{16}{|l|}{ Geographic region } \\
\hline Northeast & 632 & 8.3 & 644 & 8.5 & 0.57 & 283 & 6.9 & 275 & 6.7 & 0.77 & 690 & 6.1 & 685 & 6.1 & 0.19 \\
\hline North Central & 1,182 & 15.5 & 1,191 & 15.7 & 0.33 & 564 & 13.7 & 562 & 13.6 & 0.14 & 1,309 & 11.6 & 1,325 & 11.7 & 0.44 \\
\hline South & 3,672 & 48.3 & 3,688 & 48.5 & 0.42 & 2,271 & 55.0 & 2,284 & 55.3 & 0.63 & 6,722 & 59.6 & 6,680 & 59.2 & 0.76 \\
\hline West & 1,966 & 25.8 & 1,933 & 25.4 & 0.99 & 939 & 22.7 & 938 & 22.7 & 0.06 & 2,380 & 21.1 & 2,407 & 21.3 & 0.59 \\
\hline Other & 155 & 2.0 & 151 & 2.0 & 0.37 & 72 & 1.7 & 70 & 1.7 & 0.37 & 183 & 1.6 & 187 & 1.7 & 0.28 \\
\hline \multicolumn{16}{|l|}{ Dose } \\
\hline Standard & 5,714 & 75.1 & & & & 3,452 & 83.6 & 3,535 & 85.6 & & 8,856 & 78.5 & 8,044 & 71.3 & \\
\hline Reduced & 1,893 & 24.9 & & & & 677 & 16.4 & 594 & 14.4 & & 2,428 & 21.5 & 3,240 & 28.7 & \\
\hline \multicolumn{16}{|l|}{ Baseline comorbidity } \\
\hline $\begin{array}{l}\text { Deyo-Charlson Comorbidity } \\
\text { Index score }\end{array}$ & 2.5 & 2.4 & 2.5 & 2.4 & 1.51 & 1.92 & 2.09 & 1.92 & 2.07 & 0.05 & 2.2 & 2.3 & 2.2 & 2.3 & 0.24 \\
\hline CHADS $_{2}$ score & 2.6 & 1.4 & 2.6 & 1.4 & 1.28 & 2.2 & 1.3 & 2.2 & 1.4 & 0.73 & 2.4 & 1.4 & 2.4 & 1.4 & 0.27 \\
\hline $0=$ low risk & 422 & 5.5 & 408 & 5.4 & 0.81 & 340 & 8.2 & 348 & 8.4 & 0.70 & 675 & 6.0 & 637 & 5.6 & 1.44 \\
\hline $1=$ moderate risk & 1,318 & 17.3 & 1,294 & 17.0 & 0.84 & 1,030 & 24.9 & 1,048 & 25.4 & 1.00 & 2,344 & 20.8 & 2,362 & 20.9 & 0.39 \\
\hline $2=$ high risk & 2,247 & 29.5 & 2,360 & 31.0 & 3.23 & 1,287 & 31.2 & 1,289 & 31.2 & 0.10 & 3,513 & 31.1 & 3,555 & 31.5 & 0.80 \\
\hline$>2=$ high risk & 3,620 & 47.6 & 3,545 & 46.6 & 1.98 & 1,472 & 35.7 & 1,444 & 35.0 & 1.42 & 4,752 & 42.1 & 4,730 & 41.9 & 0.39 \\
\hline $\mathrm{CHA}_{2} \mathrm{DS}_{2}$-VASc score & 4.2 & 1.8 & 4.1 & 1.8 & 0.81 & 3.6 & 1.7 & 3.6 & 1.8 & 0.82 & 4.0 & 1.7 & 4.0 & 1.7 & 0.16 \\
\hline $0=$ low risk & 108 & 1.4 & 111 & 1.5 & 0.33 & 89 & 2.2 & 127 & 3.1 & 5.77 & 183 & 1.6 & 170 & 1.5 & 0.93 \\
\hline $1=$ moderate risk & 363 & 4.8 & 377 & 5.0 & 0.86 & 353 & 8.5 & 332 & 8.0 & 1.84 & 610 & 5.4 & 643 & 5.7 & 1.28 \\
\hline $2=$ high risk & 872 & 11.5 & 832 & 10.9 & 1.67 & 694 & 16.8 & 689 & 16.7 & 0.32 & 1,509 & 13.4 & 1,452 & 12.9 & 1.50 \\
\hline$>2=$ high risk & 6,264 & 82.3 & 6,287 & 82.6 & 0.80 & 2,993 & 72.5 & 2,981 & 72.2 & 0.65 & 8,982 & 79.6 & 9,019 & 79.9 & 0.82 \\
\hline HAS-BLED score & 3.0 & 1.3 & 3.0 & 1.3 & 1.65 & 2.8 & 1.2 & 2.8 & 1.2 & 0.14 & 3.0 & 1.2 & 3.0 & 1.2 & 0.71 \\
\hline $0=$ low risk & 100 & 1.3 & 110 & 1.4 & 1.13 & 75 & 1.8 & 84 & 2.0 & 1.59 & 139 & 1.2 & 129 & 1.1 & 0.82 \\
\hline $1-2=$ moderate risk & 2,606 & 34.3 & 2,737 & 36.0 & 3.61 & 1,782 & 43.2 & 1,704 & 41.3 & 3.83 & 4,043 & 35.8 & 4,041 & 35.8 & 0.04 \\
\hline$>2=$ high risk & 4,901 & 64.4 & 4,760 & 62.6 & 3.85 & 2,272 & 55.0 & 2,341 & 56.7 & 3.37 & 7,102 & 62.9 & 7,114 & 63.0 & 0.22 \\
\hline Baseline prior bleed & 1,525 & 20.0 & 1,484 & 19.5 & 1.35 & 606 & 14.7 & 602 & 14.6 & 0.27 & 1,903 & 16.9 & 1,907 & 16.9 & 0.09 \\
\hline Baseline prior stroke & 931 & 12.2 & 907 & 11.9 & 0.97 & 365 & 8.8 & 354 & 8.6 & 0.94 & 1,090 & 9.7 & 1,113 & 9.9 & 0.69 \\
\hline Congestive heart failure & 2,052 & 27.0 & 2,033 & 26.7 & 0.56 & 823 & 19.9 & 777 & 18.8 & 2.82 & 2,469 & 21.9 & 2,490 & 22.1 & 0.45 \\
\hline Diabetes & 2,631 & 34.6 & 2,593 & 34.1 & 1.05 & 1,303 & 31.6 & 1,313 & 31.8 & 0.52 & 3,544 & 31.4 & 3,529 & 31.3 & 0.29 \\
\hline Hypertension & 6,469 & 85.0 & 6,450 & 84.8 & 0.70 & 3,423 & 82.9 & 3,442 & 83.4 & 1.23 & 9,694 & 85.9 & 9,741 & 86.3 & 1.20 \\
\hline Renal disease & 1,852 & 24.3 & 1,839 & 24.2 & 0.40 & 654 & 15.8 & 653 & 15.8 & 0.07 & 2,170 & 19.2 & 2,202 & 19.5 & 0.72 \\
\hline Myocardial infarction & 485 & 6.4 & 479 & 6.3 & 0.32 & 226 & 5.5 & 208 & 5.0 & 1.95 & 603 & 5.3 & 622 & 5.5 & 0.74 \\
\hline $\begin{array}{l}\text { Dyspepsia or stomach } \\
\text { discomfort }\end{array}$ & 1,404 & 18.5 & 1,398 & 18.4 & 0.20 & 746 & 18.1 & 729 & 17.7 & 1.07 & 2,068 & 18.3 & 2,074 & 18.4 & 0.14 \\
\hline Peripheral vascular disease & 3,755 & 49.4 & 3,742 & 49.2 & 0.34 & 1,786 & 43.3 & 1,746 & 42.3 & 1.96 & 5,324 & 47.2 & 5,308 & 47.0 & 0.28 \\
\hline Transient ischemic attack & 599 & 7.9 & 596 & 7.8 & 0.15 & 251 & 6.1 & 257 & 6.2 & 0.60 & 824 & 7.3 & 827 & 7.3 & 0.10 \\
\hline Coronary artery disease & 3,146 & 41.4 & 3,126 & 41.1 & 0.53 & 1,512 & 36.6 & 1,478 & 35.8 & 1.71 & 4,534 & 40.2 & 4,544 & 40.3 & 0.18 \\
\hline \multicolumn{16}{|l|}{ Baseline medication use } \\
\hline $\begin{array}{l}\text { Angiotensin-converting } \\
\text { enzyme inhibitor }\end{array}$ & 2,696 & 35.4 & 2,714 & 35.7 & 0.49 & 1,383 & 33.5 & 1,411 & 34.2 & 1.43 & 3,876 & 34.3 & 3,866 & 34.3 & 0.19 \\
\hline Angiotensin receptor blocker & 1,983 & 26.1 & 1,921 & 25.3 & 1.87 & 1,126 & 27.3 & 1,126 & 27.3 & 0.00 & 3,195 & 28.3 & 3,218 & 28.5 & 0.45 \\
\hline Amiodarone & 772 & 10.1 & 765 & 10.1 & 0.31 & 365 & 8.8 & 359 & 8.7 & 0.51 & 1,119 & 9.9 & 1,127 & 10.0 & 0.24 \\
\hline
\end{tabular}


TABLE 1 Baseline Characteristics of NVAF Patients in the Propensity Score-Matched Cohorts (continued)

\begin{tabular}{|c|c|c|c|c|c|c|c|c|c|c|c|c|c|c|c|}
\hline & \multicolumn{2}{|c|}{$\begin{array}{c}\text { Apixaban } \\
\text { Cohort } \\
(\mathrm{n}=7,607)\end{array}$} & \multicolumn{2}{|c|}{$\begin{array}{c}\text { Warfarin } \\
\text { Cohort } \\
(\mathrm{n}=7,607)\end{array}$} & \multirow[b]{2}{*}{ STD $^{a}$} & \multicolumn{2}{|c|}{$\begin{array}{l}\text { Apixaban } \\
\text { Cohort } \\
(\mathbf{n}=4,129)\end{array}$} & \multicolumn{2}{|c|}{$\begin{array}{c}\text { Dabigatran } \\
\text { Cohort } \\
(\mathrm{n}=4,129)\end{array}$} & \multirow[b]{2}{*}{ STD $^{a}$} & \multicolumn{2}{|c|}{$\begin{array}{c}\text { Apixaban } \\
\text { Cohort } \\
(\mathrm{n}=11,284)\end{array}$} & \multicolumn{2}{|c|}{$\begin{array}{c}\text { Rivaroxaban } \\
\text { Cohort } \\
(\mathrm{n}=11,284) \\
\end{array}$} & \multirow[b]{2}{*}{ STD $^{\mathrm{a}}$} \\
\hline & n/Mean & $\% / S D$ & n/Mean & $\% / S D$ & & n/Mean & $\% / S D$ & n/Mean & $\% / S D$ & & n/Mean & $\% / S D$ & n/Mean & $\% / S D$ & \\
\hline \multicolumn{16}{|l|}{ Baseline medication use } \\
\hline Dronedarone & 140 & 1.8 & 151 & 2.0 & 1.06 & 211 & 5.1 & 197 & 4.8 & 1.56 & 526 & 4.7 & 521 & 4.6 & 0.21 \\
\hline Beta blockers & 5,286 & 69.5 & 5,304 & 69.7 & 0.51 & 2,894 & 70.1 & 2,865 & 69.4 & 1.53 & 8,076 & 71.6 & 8,074 & 71.6 & 0.04 \\
\hline Statins & 4,616 & 60.7 & 4,572 & 60.1 & 1.18 & 2,420 & 58.6 & 2,446 & 59.2 & 1.28 & 6,915 & 61.3 & 6,885 & 61.0 & 0.55 \\
\hline Calcium channel blockers & 3,000 & 39.4 & 3,001 & 39.5 & 0.03 & 1,602 & 38.8 & 1,655 & 40.1 & 2.63 & 4,580 & 40.6 & 4,600 & 40.8 & 0.36 \\
\hline $\mathrm{H}_{2}$-receptor antagonist & 521 & 6.8 & 521 & 6.8 & 0.00 & 240 & 5.8 & 244 & 5.9 & 0.41 & 782 & 6.9 & 773 & 6.9 & 0.31 \\
\hline Proton pump inhibitor & 2,813 & 37.0 & 2,817 & 37.0 & 0.11 & 1,558 & 37.7 & 1,545 & 37.4 & 0.65 & 4,467 & 39.6 & 4,472 & 39.6 & 0.09 \\
\hline Antiplatelets & 1,740 & 22.9 & 1,668 & 21.9 & 2.27 & 985 & 23.9 & 1,001 & 24.2 & 0.91 & 2,800 & 24.8 & 2,824 & 25.0 & 0.49 \\
\hline Baseline inpatient admission & 3,237 & 42.6 & 3,217 & 42.3 & 0.53 & 1,521 & 36.8 & 1,485 & 36.0 & 1.81 & 4,387 & 38.9 & 4,391 & 38.9 & 0.07 \\
\hline
\end{tabular}

aStandardized difference $=100$ actual standardized difference. Standardized difference $>10$ is considered significant.

$S D=$ standard deviation; $S T D=$ standardized difference

One-to-one propensity score matching (PSM) was used to balance demographics and clinical characteristics between each matched cohort (warfarin vs. apixaban, dabigatran vs. apixaban, and rivaroxaban vs. apixaban). Age, sex, U.S. geographic region, CCI score, $\mathrm{CHA}_{2} \mathrm{DS}_{2}$-VASc score, HAS-BLED score, stroke and bleeding history, comorbidities, baseline medication use, and inpatient admissions were used to calculate propensity scores for each patient using logistic regression. ${ }^{23}$ The nearest neighbor method without replacement with a caliper of 0.01 was used to match patients. Mean standardized differences were used to assess the balance of baseline patient characteristics; values $>10 \%$ were used as the threshold for statistical significance. ${ }^{24}$

Incidence rates of stroke/SE and major bleeding in PSM cohorts were calculated as the number of stroke/SE and major bleeding events, respectively, per 100 person-years. Cox proportional hazards models with robust sandwich estimates were used to compare the risk of stroke/SE and major bleeding in each of the matched cohorts. No additional variables except treatment were included in the models since all the baseline variables were balanced.

The association between treatments and the all-cause health care cost outcomes was evaluated using generalized linear models with log-link and a gamma distribution, wherein the dependent variable was costs. ${ }^{25}$ The association between treatment and the stroke/SE-related and major bleedingrelated medical costs was evaluated using 2-part models with bootstrapping, which took the cost fields with 0 values into account. ${ }^{26}$ The costs and differences in costs, 95\% confidence interval (CI), and $P$ values for each model are reported.

\section{Sensitivity Analyses}

Sensitivity analyses were conducted to test the robustness of the main results. In the first sensitivity analysis, the cohorts were stratified by dose (reduced and standard) to determine if the treatment effects were modified by dose. Patients prescribed DOACs were stratified into subgroups of standard dose (apixaban $5 \mathrm{mg}$, dabigatran $150 \mathrm{mg}$, and rivaroxaban 20 $\mathrm{mg}$ ) and reduced dose (apixaban $2.5 \mathrm{mg}$, dabigatran $75 \mathrm{mg}$, and rivaroxaban $10 \mathrm{mg}$ or $15 \mathrm{mg}$ ) based on the index dose. Each warfarin patient was assigned to 1 of the 2 subgroups per the dose of the matched apixaban patient. In each subgroup, the balance of baseline characteristics was evaluated; when imbalance was detected (standardized difference $>10 \%$ ), the variable was included in the multivariate model. The statistical significance of the interaction between treatment and dose was evaluated.

Second, patients who had catheter ablation within 2 months before the index prescription and those who had cardioversion 1 month before or after the index drug initiation date were excluded, as they likely had a low risk of stroke/SE and were administered an OAC for their procedure, not for long-term stroke prevention. ${ }^{27,28}$

Third, a sensitivity analysis for the 6 months postindex date as follow-up was also conducted. In this analysis, patients were censored at OAC discontinuation, switch, death, disenrollment date, end of the study period (September 30, 2015), or 6 months after the index date, whichever occurred the earliest. Sensitivity analysis allowed for the follow-up period to be more balanced between the cohorts. Finally, a sensitivity analysis using the intent-to-treat method was implemented, where patient data were assessed based on the index drug regardless of discontinuation or switch.

\section{Results}

\section{Baseline Characteristics}

A total of 41,001 patients were eligible for the study, including 9,255 (22.6\%) warfarin, 4,312 (10.5\%) dabigatran, 15,680 
Real-World Comparative Effectiveness, Safety, and Health Care Costs of Oral Anticoagulants in Nonvalvular Atrial Fibrillation Patients in the U.S. Department of Defense Population

FIGURE 2 Risk of Stroke/SE and Major Bleeding in the Propensity Score-Matched Populations

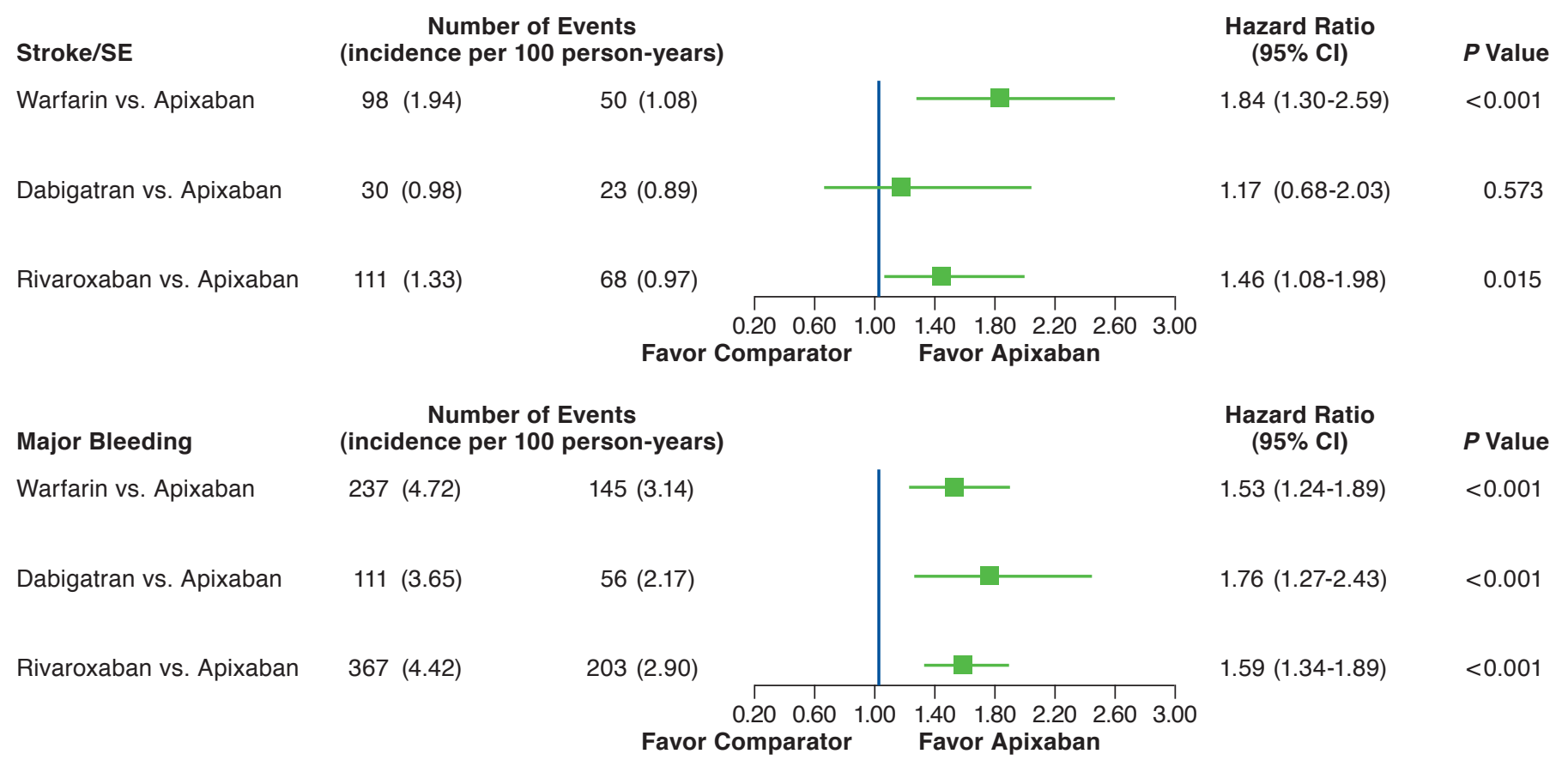

$C I=$ confidence interval; $S E=$ systemic embolism.

(38.2\%) rivaroxaban, and 11,754 (28.7\%) apixaban patients. Patients initiating apixaban were younger and had lower baseline mean $\mathrm{CHA}_{2} \mathrm{DS}_{2}$-VASc, HAS-BLED, and CCI scores than warfarin patients. However, compared with those initiating dabigatran and rivaroxaban, patients initiating apixaban were older and had higher baseline mean $\mathrm{CHA}_{2} \mathrm{DS}_{2}$-VASc, HASBLED, and CCI scores.

After 1:1 PSM, there were 7,607 warfarin-apixaban, 4,129 dabigatran-apixaban, and 11,284 rivaroxaban-apixaban matched pairs (Figure 1). Baseline demographic and clinical characteristics were balanced between the matched cohorts with standardized difference $\leq 10 \%$. Warfarin-apixaban, dabigatran-apixaban, and rivaroxaban-apixaban cohorts had mean ages of 77, 73, and 75 years, respectively. Across the 3 matched cohorts, the mean $\mathrm{CHA}_{2} \mathrm{DS}_{2}$-VASc score was 3.6-4.2, and the mean HAS-BLED score was 2.8-3.0 (Table 1). Patients were followed for a mean of 7-9 months.

\section{Risk of Stroke/SE and Major Bleeding}

The incidence rates of stroke/SE and major bleeding are shown in Figure 2. Warfarin (hazard ratio $[\mathrm{HR}]=1.84 ; 95 \% \mathrm{CI}=1.30-2.59$; $P<0.001)$ and rivaroxaban $(\mathrm{HR}=1.46 ; 95 \% \mathrm{CI}=1.08-1.98$; $P=0.015)$ were associated with a significantly higher risk of stroke/SE compared with apixaban. Dabigatran $(\mathrm{HR}=1.17$;
95\% CI $=0.68-2.03 ; P=0.573)$ patients had a nonstatistically significant higher risk of stroke/SE compared with apixaban (Figure 2).

Warfarin $(\mathrm{HR}=1.53 ; 95 \% \mathrm{CI}=1.24-1.89 ; \mathrm{P}<0.001)$, dabiga$\operatorname{tran}(\mathrm{HR}=1.76 ; 95 \% \mathrm{CI}=1.27-2.43 ; \mathrm{P}<0.001)$, and rivaroxaban $(\mathrm{HR}=1.59 ; 95 \% \mathrm{CI}=1.34-1.89 ; P<0.001)$ were associated with a significantly higher risk of major bleeding compared with apixaban (Figure 2).

\section{Health Care Costs}

Warfarin initiation was associated with similar all-cause PPPM outpatient costs ( $\$ 1,157$ vs. $\$ 1,206 ; P=0.341)$, significantly higher all-cause inpatient ( $\$ 1,092$ vs. $\$ 711 ; P=0.002)$ and medical (inpatient and outpatient; $\$ 2,249$ vs. $\$ 1,917$; $P=0.026)$ costs, and significantly lower pharmacy costs $(\$ 248$ vs. $\$ 360 ; P<0.001)$ compared with apixaban. The total allcause health care costs $(\$ 2,498$ vs. $\$ 2,277 ; P=0.148)$ were higher for warfarin than apixaban patients but were not statistically significant. Dabigatran initiation was associated with significantly higher inpatient costs ( $\$ 781$ vs. $\$ 565 ; P=0.034$ ), but numerically higher total all-cause health care costs $(\$ 2,372$ vs. $\$ 2,143 ; P=0.150)$ compared with apixaban. Rivaroxaban was associated with significantly higher inpatient (\$939 vs. \$658; $P<0.001$ ), medical (inpatient and outpatient; $\$ 2,156$ vs. 


\section{TABLE 2 Comparisons of All-Cause Health Care Costs After PSM}

\begin{tabular}{|c|c|c|c|c|c|c|c|c|c|}
\hline \multirow[b]{2}{*}{ PPPM Costs } & \multirow{2}{*}{$\begin{array}{c}\text { Apixaban } \\
\text { Cohort } \\
\mathbf{n}=7,607 \\
\text { Marginal } \\
\text { Effect }^{\text {a }}(\$)\end{array}$} & \multicolumn{2}{|c|}{$\begin{array}{l}\text { Warfarin Cohort } \\
\qquad \mathbf{n}=7,607\end{array}$} & \multirow{2}{*}{$\begin{array}{c}\text { Apixaban } \\
\text { Cohort } \\
\mathbf{n}=4,129 \\
\text { Marginal } \\
\text { Effect (\$) }\end{array}$} & \multicolumn{2}{|c|}{$\begin{array}{l}\text { Dabigatran Cohort } \\
\qquad \mathbf{n}=4,129\end{array}$} & \multirow{2}{*}{$\begin{array}{c}\text { Apixaban } \\
\text { Cohort } \\
\mathrm{n}=11,284\end{array}$} & \multicolumn{2}{|c|}{$\begin{array}{c}\text { Rivaroxaban Cohort } \\
\mathbf{n}=11,284\end{array}$} \\
\hline & & $\begin{array}{l}\text { Marginal } \\
\text { Effect (\$) }\end{array}$ & $P$ Value & & $\begin{array}{l}\text { Marginal } \\
\text { Effect (\$) }\end{array}$ & $P$ Value & & $\begin{array}{l}\text { Marginal } \\
\text { Effect (\$) }\end{array}$ & $P$ Value \\
\hline All-cause outpatient medical costs & 1,206 & 1,157 & 0.341 & 1,222 & 1,236 & 0.888 & 1,174 & 1,217 & 0.284 \\
\hline All-cause inpatient medical costs & 711 & 1,092 & 0.002 & 565 & 781 & 0.034 & 658 & 939 & $<0.001$ \\
\hline All-cause medical costs & 1,917 & 2,249 & 0.026 & 1,787 & 2,018 & 0.143 & 1,832 & 2,156 & $<0.001$ \\
\hline Pharmacy costs & 360 & 248 & $<0.001$ & 356 & 354 & 0.904 & 368 & 390 & 0.032 \\
\hline All-cause health care costs & 2,277 & 2,498 & 0.148 & 2,143 & 2,372 & 0.150 & 2,200 & 2,546 & $<0.001$ \\
\hline
\end{tabular}

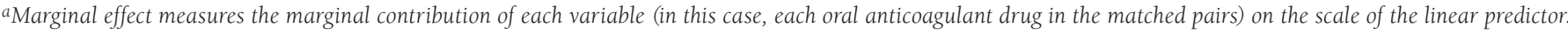
PPPM = per patient per month; PSM= propensity score matching.

$\$ 1,832 ; P<0.001$ ), pharmacy ( $\$ 390$ vs. $\$ 368 ; P=0.032$ ), and total health care costs $(\$ 2,546$ vs. $\$ 2,200, P<0.001)$ compared with apixaban (Table 2).

Warfarin patients had significantly higher PPPM stroke/ SE-related medical costs ( $\$ 118$ vs. $\$ 46 ; P=0.012$ ) compared with apixaban patients. Dabigatran ( $\$ 61$ vs. $\$ 32 ; P=0.240$ ) and rivaroxaban ( $\$ 58$ vs. $\$ 38 ; P=0.057$ ) patients had numerically higher stroke/SE-related medical costs compared with apixaban. Warfarin ( $\$ 166$ vs. $\$ 76 ; P=0.003$ ), dabigatran ( $\$ 114$ vs. $\$ 58 ; P=0.025)$, and rivaroxaban ( $\$ 137$ vs. $\$ 69 ; P<0.001$ ) patients had significantly higher PPPM major bleeding-related medical costs compared with apixaban patients (Figure 3).

\section{Sensitivity Analyses}

The sensitivity analyses were generally consistent with the primary study results. A significant interaction was found for the dose of rivaroxaban and apixaban for major bleeding $(P=0.039)$. Standard-dose rivaroxaban was associated with a significantly higher risk of major bleeding ( $H R=1.81$; $95 \% \mathrm{CI}=1.47-2.24)$ compared with standard-dose apixaban, whereas reduced-dose rivaroxaban was associated with a similar risk of major bleeding ( $\mathrm{HR}=1.23 ; 95 \% \mathrm{CI}=0.91-1.67)$ compared with reduced-dose apixaban (Appendix A, available in online article). The other sensitivity analyses showed results similar to the results of the main analysis (Appendix B, available in online article).

\section{Discussion}

In this real-world study of NVAF patients initiating OAC treatment in the U.S. DoD population, warfarin and rivaroxaban were associated with a significantly higher risk of stroke/ SE and major bleeding compared with apixaban. Dabigatran initiation was associated with a numerically higher risk of stroke/SE and a significantly higher risk of major bleeding compared with apixaban. Correspondingly, stroke/SE-related medical costs were significantly higher for warfarin patients compared with apixaban. Major bleeding-related medical costs were significantly higher for those prescribed warfarin, dabigatran, and rivaroxaban compared with apixaban. Rivaroxaban was associated with significantly higher total all-cause health care costs compared with apixaban. Warfarin and dabigatran patients had similar total all-cause health care costs compared with apixaban. The robustness of the clinical results was tested by several sensitivity analyses, which were consistent with the main analysis.

The results of this observational study are generally consistent with clinical trials and real-world studies with a focus on apixaban. In the ARISTOTLE trial, apixaban treatment was superior to warfarin in reducing the risk of stroke/SE $(\mathrm{HR}=0.79 ; 95 \% \mathrm{CI}=0.66-0.95 ; P=0.011)$ with fewer major bleeding events ( $\mathrm{HR}=0.69 ; 95 \% \mathrm{CI}=0.60-0.80 ; P<0.001)^{7}$ Our study also demonstrated the same associations between apixaban and warfarin for the risk of stroke/SE and major bleeding.

No head-to-head clinical trials are available for DOAC comparisons. Indirect DOAC comparisons using clinical trial data showed that apixaban had a similar risk of stroke/SE compared with rivaroxaban and reduced- or standard-dose dabigatran and a significantly lower risk of major bleeding compared with rivaroxaban and standard-dose dabigatran. ${ }^{29}$ In addition, few real-world studies examined the risk of stroke/SE and major bleeding among apixaban, dabigatran, and rivaroxaban. In the Noseworthy et al. study (2016), no significant differences were found regarding the risk of stroke/SE for patients prescribed apixaban, dabigatran, or rivaroxaban; however, apixaban use was associated with a 50\% lower risk of major bleeding compared with dabigatran and a $61 \%$ lower risk of major bleeding compared with rivaroxaban. ${ }^{11}$ Similarly, in the Amin et al. study (2018) using OptumInsight data, dabigatran and rivaroxaban were associated with a similar risk of stroke/SE but a significantly higher risk of major bleeding compared with apixaban. ${ }^{15}$ Our study showed consistent results except that 

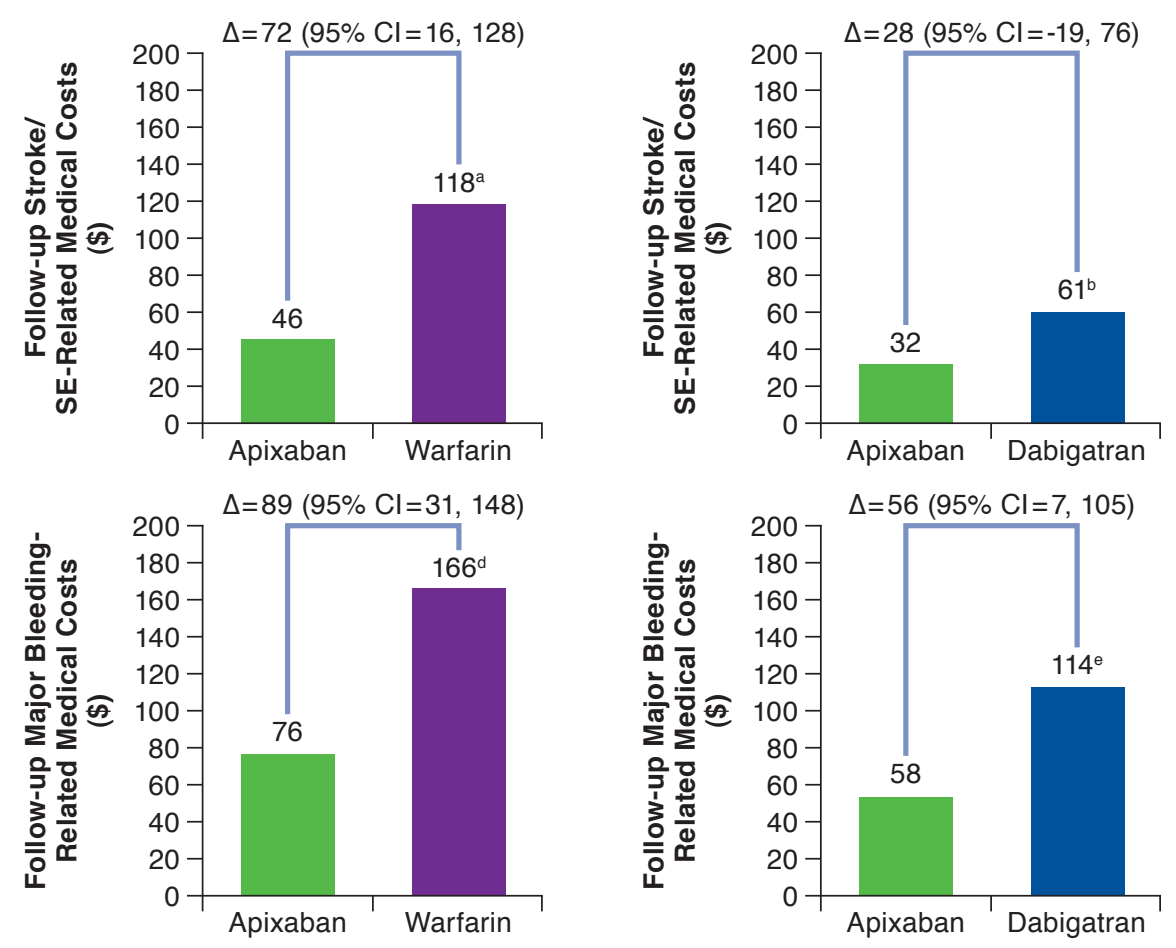
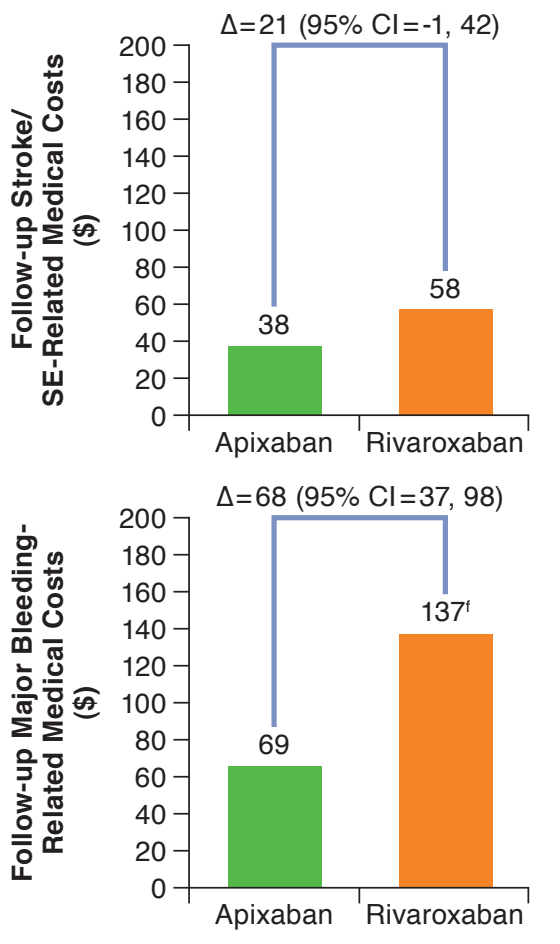

$a P=0.012$

$b_{P}=0.240$

$c P=0.057$

$d P=0.003$

${ }^{e} P=0.025$

$f_{P}<0.001$

$\Delta$ =differences in costs; $C I=$ confidence interval; $P P P M=$ per patient per month; SE= systemic embolism.

rivaroxaban was associated with a significantly higher risk of stroke/SE compared with apixaban. This may be because of the different patient population across studies. For example, this DoD population had a higher mean age compared with the populations in the other studies.

In real-world studies comparing the effectiveness and safety between apixaban and warfarin, apixaban showed consistent results of significantly lower risks of stroke/SE and major bleeding compared with warfarin. ${ }^{15,19,30-32}$ Several studies using the clinical trials data and Markov decision models have shown that DOACs are cost-effective alternatives to warfarin, with apixaban providing the greatest cost-effectiveness compared with dabigatran, rivaroxaban, and warfarin..$^{33-35}$ However, limited real-world data are available regarding comparative health care costs for OACs. The all-cause medical cost results found here are similar to previous studies. ${ }^{15,34}$ For example, in the Deitelzweig et al. (2016) evaluation of DOACs using Premier data, rivaroxaban patients incurred significantly higher all-cause hospitalization readmission costs (difference $=\$ 413 ; P=0.003$ ) compared with apixaban patients. ${ }^{14}$ Also, in Amin et al., apixaban was associated with significantly lower all-cause health care costs PPPM and major bleeding-related medical costs compared with warfarin and significantly lower major bleeding-related medical costs compared with dabigatran. ${ }^{15}$ Our study showed similar total all-cause health care costs between warfarin and apixaban patients; however, in a study using Medicare data, warfarin patients were found to have significantly higher all-cause health care costs compared with apixaban patients. ${ }^{34}$ The difference in results may be because of the potential underestimation of pharmacy costs in the DoD data. As more longitudinal data become available and as treatment practices change over time, additional studies are warranted regarding the comparative economic outcomes between OACs among NVAF patients. 


\section{Limitations}

This study has several limitations. Because of the nature of claims studies, diagnoses and procedures in this study were identified using ICD-9-CM, Current Procedural Terminology, and Healthcare Common Procedure Coding System codes and National Drug Code numbers. These coding systems were originally designed for billing purposes rather than research. For example, the presence of a claim for a filled prescription does not indicate whether the medication was consumed or taken as prescribed, and no international normalized ratio value is available for the prescription of warfarin. Moreover, the pharmacy costs in military facilities are not captured in the DoD data. Only the TRICARE Mail Order Pharmacy and retail pharmacy expenditures are available, which may have caused the underestimation of the pharmacy cost calculation in our analysis.

Although most patients (>85\%) in our study had Medicare as their primary insurance, all medical claims and costs should have been included in the DoD data. For patients with Medicare insurance, the DoD pays for any costs that are not covered by Medicare in military and civilian medical facilities, with the complete cost information in the data. ${ }^{36}$

Also, although PSM was used with the cohorts, potential residual confounders existed, so no causal inferences could be drawn. In addition, PSM was conducted between each matched pair; thus, 1 patient may have been included in multiple comparisons, so no conclusions could be drawn across the matched cohorts.

Although no direct comparison to the clinical trials can be made given the nature of retrospective observational studies, our findings from the main analysis, as well as subgroup and sensitivity analyses, provided additional real-world evidence and support for the clinical trial study results. Finally, only treatment-naive patients and patients in the DoD population were studied, which may have affected the generalization of the study results.

\section{Conclusions}

Using U.S. DoD data, this study adds real-world evidence to help compare the effectiveness, safety, and health care costs of apixaban to warfarin, dabigatran, and rivaroxaban. Among NVAF patients, warfarin and rivaroxaban were associated with a significantly higher risk of stroke/SE and major bleeding when compared with apixaban. Dabigatran use was associated with a numerically higher risk of stroke/SE and a significantly higher risk of major bleeding compared with apixaban.

Warfarin patients incurred significantly higher stroke/ SE-related, major bleeding-related, and all-cause medical costs compared with apixaban patients. Dabigatran and rivaroxaban patients incurred numerically higher stroke/SE-related medical costs and significantly higher major bleeding-related medical costs compared with apixaban patients. Total all-cause health care costs were similar between patients prescribed dabigatran and apixaban but were significantly higher for those prescribed rivaroxaban compared with apixaban.

This observational study supplements the clinical trial findings on the effectiveness and safety of apixaban relative to warfarin. In addition, it provides evidence on the clinical and economic comparisons of apixaban with other DOACs, which will potentially facilitate the decision-making process in the prevention of stroke/SE among NVAF patients.

\section{Authors}

KIRAN GUPTA, PhD; LISA ROSENBLATT, MD; SHALINI HEDE, PharmD; and ANAGHA NADKARNI, PhD, Bristol-Myers Squibb, Lawrenceville, New Jersey. JEFFREY TROCIO, MPH; OLUWASEYI DINA, MPH; JACK MARDEKIAN, PhD; and TOM SHANK, PharmD, Pfizer, New York City, New York. XIANCHEN LIU, MD, PhD, Pfizer, New York City, New York, and the University of Tennessee Health Science Center, Memphis. ALLISON KESHISHIAN, MPH, and QISU ZHANG, MPH, STATinMED Research, Ann Arbor, Michigan.

AUTHOR CORRESPONDENCE: Allison Keshishian, MPH, STATinMED Research, 211 N. 4th Ave., \#2B, Ann Arbor, MI 48105. Tel.: 734.222.5426; E-mail: akeshishian@statinmed.com.

\section{DISCLOSURES}

This study was funded by Bristol-Myers Squibb and Pfizer, which were involved in the study design, as well as in the writing and revision of the manuscript. Keshishian and Zhang are paid employees of STATinMED Research, which was paid by Bristol-Myers Squibb and Pfizer to conduct this study and develop the manuscript. Gupta, Rosenblatt, Hede, and Nadkarni are paid employees of Bristol-Myers Squibb. Trocio, Dina, Mardekian, Liu, and Shank are paid employees of Pfizer.

\section{REFERENCES}

1. Wolf PA, Abbott RD, Kannel WB. Atrial fibrillation as an independent risk factor for stroke: the Framingham Study. Stroke. 1991;22(8):983-88.

2. Wolf PA, Mitchell JB, Baker CS, Kannel WB, D’Agostino RB. Impact of atrial fibrillation on mortality, stroke, and medical costs. Arch Intern Med. 1998;158(3):229-34.

3. Go AS, Hylek EM, Phillips KA, et al. Prevalence of diagnosed atrial fibrillation in adults: national implications for rhythm management and stroke prevention: the AnTicoagulation and Risk Factors in Atrial Fibrillation (ATRIA) Study. JAMA. 2001;285(18):2370-75.

4. Coyne KS, Paramore C, Grandy S, Mercader M, Reynolds M, Zimetbaum P. Assessing the direct costs of treating nonvalvular atrial fibrillation in the United States. Value Health. 2006;9(5):348-56.

5. January CT, Wann LS, Alpert JS, et al. 2014 AHA/ACC/HRS guideline for the management of patients with atrial fibrillation: executive summary: a report of the American College of Cardiology/American Heart Association Task Force on practice guidelines and the Heart Rhythm Society. Circulation. 2014;130(23):2071-104

6. Hart RG, Pearce LA, Aguilar MI. Meta-analysis: antithrombotic therapy to prevent stroke in patients who have nonvalvular atrial fibrillation. Ann Intern Med. 2007;146(12):857-67. 


\section{Real-World Comparative Effectiveness, Safety, and Health Care Costs of Oral Anticoagulants in Nonvalvular Atrial Fibrillation Patients in the U.S. Department of Defense Population}

7. Connolly SJ, Ezekowitz MD, Yusuf S, et al. Dabigatran versus warfarin in patients with atrial fibrillation. N Engl J Med. 2009;361(12):1139-51.

8. Granger CB, Alexander JH, McMurray JJ, et al. Apixaban versus warfarin in patients with atrial fibrillation. N Engl J Med. 2011;365(11):981-92.

9. Patel MR, Mahaffey KW, Garg J, et al. Rivaroxaban versus warfarin in nonvalvular atrial fibrillation. N Engl J Med. 2011;365(10):883-91.

10. Giugliano RP, Ruff CT, Braunwald E, et al. Edoxaban versus warfarin in patients with atrial fibrillation. N Engl J Med. 2013;369(22):2093-104

11. Noseworthy PA, Yao X, Abraham NS, Sangaralingham LR, McBane RD, Shah ND. Direct comparison of dabigatran, rivaroxaban, and apixaban for effectiveness and safety in nonvalvular atrial fibrillation. Chest. 2016;150(6):1302-12

12. Bai Y, Shi XB, Ma CS, Lip GYH. Meta-analysis of effectiveness and safety of oral anticoagulants in atrial fibrillation with focus on apixaban. Am J Cardiol. 2017;120(9):1689-95.

13. Xie L, Vo L, Keshishian A, et al. Comparison of hospital length of stay and hospitalization costs among patients with non-valvular atrial fibrillation treated with apixaban or warfarin: an early view. J Med Econ. 2016;19(8):769-76.

14. Deitelzweig S, Bruno A, Trocio J, et al. An early evaluation of bleedingrelated hospital readmissions among hospitalized patients with nonvalvular atrial fibrillation treated with direct oral anticoagulants. Curr Med Res Opin. 2016;32(3):573-82

15. Amin A, Keshishian A, Vo L, et al. Real-world comparison of all-cause hospitalizations, hospitalizations due to stroke and major bleeding, and costs for non-valvular atrial fibrillation patients prescribed oral anticoagulants in a U.S. health plan. J Med Econ. 2018;21(3):244-53.

16. Office of the Assistant Secretary of Defense for Health Affairs; TRICARE Management Activity. Guide for DoD researchers on using MHS data. October 10, 2012. Available at: https://www.health.mil/Reference-Center/ Publications/2012/10/10/Guide-for-DoD-Researchers-on-Using-MHS-Data. Accessed August 13, 2018

17. Jensen PN, Johnson K, Floyd J, Heckbert SR, Carnahan R, Dublin S. Identifying atrial fibrillation from electronic medical data: a systematic review. Pharmacoepidemiol Drug Saf. 2012;21(Suppl 1):141-47.

18. Teutsch C, Huisman MV, Lip GYH, et al. Persistence with dabigatran therapy for stroke prevention in patients with non-valvular atrial fibrillation: the Gloria-AF Registry. Blood. 2016;128(22):2616.

19. Yao X, Abraham NS, Sangaralingham LR, et al. Effectiveness and safety of dabigatran, rivaroxaban, and apixaban versus warfarin in nonvalvular atrial fibrillation. J Am Heart Assoc. 2016;5(6):e003725.

20. Cunningham A, Stein CM, Chung CP, Daugherty JR, Smalley WE, Ray WA. An automated database case definition for serious bleeding related to oral anticoagulant use. Pharmacoepidemiol Drug Saf. 2011;20(6):560-66.

21. Berger ML, Mamdani M, Atkins D, Johnson ML. Good research practices for comparative effectiveness research: defining, reporting and interpreting nonrandomized studies of treatment effects using secondary data sources: the ISPOR Good Research Practices for Retrospective Database Analysis Task Force Report_Part I. Value Health. 2009;12(8):1044-52.
22. Willke RJ, Mullins CD. "Ten commandments" for conducting comparative effectiveness research using "real-world data." J Manag Care Pharm. 2011;17(9 Suppl A):S10-15. Available at: https://www.jmcp.org/doi/10.18553/ jmcp.2011.17.s9-a.S10.

23. Austin PC. The use of propensity score methods with survival or timeto-event outcomes: reporting measures of effect similar to those used in randomized experiments. Stat Med. 2014;33(7):1242-58.

24. Austin PC. Balance diagnostics for comparing the distribution of baseline covariates between treatment groups in propensity-score matched samples. Stat Med. 2009;28(25):3083-107.

25. Blough DK, Ramsey SD. Using generalized linear models to assess medical care costs. Health Serv Outcomes Res Methodol. 2000;1(2):185-202.

26. Lachenbruch PA. Comparisons of two-part models with competitors. Stat Med. 2001;20(8):1215-34.

27. Oketani N, Nademanee K. Mortality and stroke reduction after successful catheter ablation for high-risk patients with atrial fibrillation. US Cardiology. 2008;5(1):54-56.

28. Garg A, Khunger M, Seicean S, Chung MK, Tchou PJ. Incidence of thromboembolic complications within 30 days of electrical cardioversion performed within 48 hours of atrial fibrillation onset. JACC Clin Electrophysiol. 2016;2(4):487-94.

29. Lip GY, Larsen TB, Skjøth F, Rasmussen LH. Indirect comparisons of new oral anticoagulant drugs for efficacy and safety when used for stroke prevention in atrial fibrillation. J Am Coll Cardiol. 2012;60(8):738-46.

30. Lip GY, Keshishian A, Kamble S, et al. Real-world comparison of major bleeding risk among non-valvular atrial fibrillation patients initiated on apixaban, dabigatran, rivaroxaban, or warfarin: a propensity score matched analysis. Thromb Haemost. 2016;116(5):975-86.

31. Li XS, Deitelzweig S, Keshishian A, et al. Effectiveness and safety of apixaban versus warfarin in non-valvular atrial fibrillation patients in "real-world" clinical practice: a propensity-matched analysis of 76,940 patients. Thromb Haemost. 2017;117(6):1072-82

32. Amin A, Keshishian A, Trocio J, et al. Risk of stroke/systemic embolism, major bleeding and associated costs in non-valvular atrial fibrillation patients who initiated apixaban, dabigatran or rivaroxaban compared with warfarin in the United States Medicare population. Curr Med Res Opin. 2017;33(9):1595-604

33. Coyle D, Coyle K, Cameron C, et al. Cost-effectiveness of new oral anticoagulants compared with warfarin in preventing stroke and other cardiovascular events in patients with atrial fibrillation. Value Health. 2013;16(4):498-506

34. Canestaro WJ, Patrick AR, Avorn J, et al. Cost-effectiveness of oral anticoagulants for treatment of atrial fibrillation. Circ Cardiovasc Qual Outcomes. 2013;6(6):724-31.

35. Kamel H, Easton JD, Johnston SC, Kim AS. Cost-effectiveness of apixaban vs warfarin for secondary stroke prevention in atrial fibrillation. Neurology. 2012;79(14):1428-34.

36. Tamayo S, Frank Peacock W, Patel M, et al. Characterizing major bleeding in patients with nonvalvular atrial fibrillation: a pharmacovigilance study of 27,467 patients taking rivaroxaban. Clin Cardiol. 2015;38(2):63-68 
Real-World Comparative Effectiveness, Safety, and Health Care Costs of Oral Anticoagulants

in Nonvalvular Atrial Fibrillation Patients in the U.S. Department of Defense Population

APPENDIX A Dose Sensitivity Analysis for Propensity Score-Matched Patients

\begin{tabular}{|c|c|c|c|c|c|c|}
\hline & $\begin{array}{c}\text { Warfarin vs. } \\
\text { Apixaban }\end{array}$ & $P$ Value ${ }^{a}$ & $\begin{array}{c}\text { Dabigatran vs. } \\
\text { Apixaban }\end{array}$ & $P$ Value ${ }^{\mathrm{a}}$ & $\begin{array}{l}\text { Rivaroxaban vs. } \\
\text { Apixaban }\end{array}$ & $P$ Value ${ }^{a}$ \\
\hline \multicolumn{7}{|c|}{ Stroke/SE, HR (95\% CI) } \\
\hline Reduced doseb & $1.39(0.72-2.67)$ & \multirow{2}{*}{0.315} & $0.82(0.29-2.28)$ & \multirow{2}{*}{0.409} & $1.42(0.83-2.42)$ & \multirow{2}{*}{0.911} \\
\hline Standard dose $e^{c}$ & $2.06(1.37-3.10)$ & & $1.37(0.71-2.65)$ & & $1.47(1.01-2.13)$ & \\
\hline \multicolumn{7}{|c|}{ Major bleeding, HR (95\% CI) } \\
\hline Reduced doseb & $1.52(1.06-2.19)$ & \multirow{2}{*}{0.803} & $1.89(1.00-3.57)$ & \multirow{2}{*}{0.763} & $1.23(0.91-1.67)$ & \multirow{2}{*}{0.039} \\
\hline Standard dosec & $1.61 \quad(1.24-2.09)$ & & $1.69(1.16-2.47)$ & & $1.81 \quad(1.47-2.24)$ & \\
\hline
\end{tabular}

aP value is for interaction.

${ }^{b}$ Reduced dose: $2.5 \mathrm{mg}$ apixaban, $75 \mathrm{mg}$ dabigatran, $10 \mathrm{mg}$ or $15 \mathrm{mg}$ rivaroxaban.

cStandard dose: $5 \mathrm{mg}$ apixaban, $150 \mathrm{mg}$ dabigatran, $20 \mathrm{mg}$ rivaroxaban.

$C I=$ confidence interval; $H R=$ hazard ratio; $S E=$ systemic embolis

\section{APPENDIX B Sensitivity Analyses for Propensity Score-Matched Patients}

\begin{tabular}{|c|c|c|c|c|c|c|}
\hline & $\begin{array}{c}\text { Warfarin vs. } \\
\text { Apixaban }\end{array}$ & $P$ Value & $\begin{array}{c}\text { Dabigatran vs. } \\
\text { Apixaban }\end{array}$ & $P$ Value & $\begin{array}{c}\text { Rivaroxaban vs. } \\
\text { Apixaban }\end{array}$ & $P$ Value \\
\hline \multicolumn{7}{|c|}{ Excluding patients with catheter ablation or cardioversion, HR (95\% CI) } \\
\hline Stroke/SE & $1.84(1.03-2.59)$ & $<0.001$ & $1.17(0.68-2.03)$ & 0.573 & $1.46(1.08-1.98)$ & 0.015 \\
\hline Major bleeding & $1.53(1.24-1.89)$ & $<0.001$ & $1.76(1.27-2.43)$ & $<0.001$ & $1.59(1.34-1.89)$ & $<0.001$ \\
\hline \multicolumn{7}{|c|}{ Censoring at 6 months, HR (95\% CI) } \\
\hline Stroke/SE & $1.95(1.26-3.01)$ & 0.003 & $1.14 \quad(0.58-2.23)$ & 0.711 & $1.64(1.13-2.39)$ & 0.009 \\
\hline Major bleeding & $1.70 \quad(1.31-2.22)$ & $<0.001$ & $1.81 \quad(1.18-2.77)$ & 0.006 & $1.85(1.48-2.32)$ & $<0.001$ \\
\hline \multicolumn{7}{|c|}{ Intent to treat, HR $(95 \% \mathrm{CI})$} \\
\hline Stroke/SE & $1.58(1.23-2.04)$ & $<0.001$ & $1.18 \quad(0.82-1.69)$ & 0.387 & $1.57(1.25-1.96)$ & $<0.001$ \\
\hline Major bleeding & $1.34(1.14-1.57)$ & $<0.001$ & $1.35(1.06-1.71)$ & 0.015 & $1.38(1.20-1.58)$ & $<0.001$ \\
\hline
\end{tabular}

